\title{
LED Illumination System with Color Rendering Functionality to Improve the Comfort of Indoor Spaces
}

\author{
Kohei Fujimoto ${ }^{\mathrm{a}}$, Keiji Matsumoto $\mathrm{a}^{\mathrm{a}^{*}}$

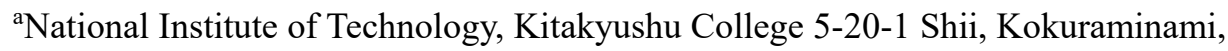 \\ Kitakyushu, Fukuoka, 802-0985, Japan \\ *Corresponding Author:kmatsu@kct.ac.jp
}

\begin{abstract}
In this study, we propose an light-emitting diode (LED) illumination system that automatically estimates human comfort by capturing and analyzing brainwaves in real time and changes the brightness and color of an LED lamp accordingly to improve comfort. Taking system feasibility into account, we applied a single-channel electroencephalogram (EEG) sensor to measure brainwaves. Human comfort is estimated using the Index of Brain Resting State (IBRS), which is calculated by analyzing each frequency element of measured brainwaves. The luminosity of red, green, and blue LEDs is variously adjusted by the system to find a calming color for the person. The most calming color is estimated using the average and standard deviation of the IBRS calculated from the measured brainwaves.
\end{abstract}

Keywords: illumination, LED, EEG, control system.

\section{Introduction}

Light-emitting diode (LED) lamps have been used in practical display applications since their development in the 1960s. Subsequently, the development of a white LED was sparked by the advent of the blue LED. Because LEDs are environmentally friendly, conserve energy, and last a long time, LED lamps are being used to replace conventional incandescent lamps. Moreover, LEDs mimic natural light spectra more closely than fluorescent lights, and are thought of as the fourth-generation light. There are a lot of reports related to LED development. Because many white LEDs are a combination of blue light from blue LEDs and yellow light from yellow fluorescent material, they are inferior to incandescent lamps in terms of their red component. In response to this, research is being carried out to add red, green, and blue LEDs to white LEDs to achieve a comfortable lighting environment $[1,2]$. In terms of the influence of the chromatic color, it is known that people tend to become excited when exposed to the color red and become calm when exposed to blue [3]. The psychological and physiological effects of using chromatic color as a light source are also being researched $[4,5]$. According to these studies, although the psychological effects of chromatic colors can be observed to some extent, the physiological effects were not clearly confirmed. In another study, a system with an LED lamp was researched that had color and brightness controls based on the outside environment $[6,7]$. The circadian rhythm of the human body functions such that body temperature, blood pressure, and hormone secretions fluctuate with the movement of the sun. This research aims to increase comfort and productivity by using an LED lamp to simulate sunlight in the workplace. Many studies on LED lamps have been reported and all of them take comfort into account in some way; however, it is difficult to determine the comfort of an individual because illumination preference varies widely from person to person.

In this study, we propose an LED illumination system that automatically estimates human comfort by capturing and analyzing brainwaves in real time and changes the brightness and color of an LED lamp accordingly to improve comfort. Taking system feasibility into account, we used a singlechannel electroencephalogram (EEG) sensor to measure brainwaves. Human comfort is estimated using the Index of Brain Resting State (IBRS) calculated by analyzing each frequency element of the measured brainwaves. If a system is realized that can provide a comfortable light source in 
harmony with the day, it can be used to help people relieve stress.

\section{Method}

\subsection{EEG device}

Traditional EEG experiments are usually impractical in real-world settings because they often use multi-channel electrodes for accurate measurement. For this reason, it seems difficult to realize the applied product of EEG feature using it easily. In this experiment, a single-channel dry EEG sensor manufactured by NeuroSky Inc. placed on the forehead is used as a user-friendly interface. The singlechannel EEG sensor has two electrodes to measure brain potentials; an active electrode and a reference electrode. The EEG sensor is worn on the head of the test subject, and it monitors the electrical potential between the electrodes attached on the forehead (active) and the earlobe (reference). It is possible to analyze each frequency elements in detail, because the EEG signals are presented at every $0.25 \mathrm{~Hz}$ in the range of 0 to 255 frequency band [8].

\subsection{Index of Brain Resting State (IBRS)}

The IBRS, which is the electroencephalographic index to evaluate the resting state of the human, was proposed by Nakamura et al [9]. Since then, the IBRS has been used for various kinds of research as a human condition estimation [10-12]. Figure.1 shows the conceptual diagram of the human state and brainwave frequencies. Generally, the alpha band is electroencephalographically used as an index of rest, using its quantity of magnitude as a discrimination criterion. However, further brainwave information is required for correct estimation of the human state because the alpha band is considerably affected by visual information [13]. Generally, the beta band components increases in amplitudes and its frequencies change into fast wave oscillations if the brain enters an aroused and excited state. Conversely, when the brain becomes drowsy and enters a suppressed state, the theta band components increases in amplitudes and its frequencies change into slow wave oscillations. The condition between arousal and drowsiness is thought as the brain's resting state. Therefore, the magnitude of the alpha band compared to the theta and beta bands is defined as the IBRS. The mathematical definition of the IBRS is given in equations (1) and (2). The IBRS is visualized in two dimensions using the ratio between the theta wave and the alpha wave or between the beta wave and the alpha wave (Fig.2). The horizontal axis is defined as the IBRS's $x$ component, represented by both the theta wave and alpha wave, while the vertical axis is defined as the IBRS's $y$ component, represented by both the beta wave and the alpha wave. Both the IBRS's components are represented in a range from -1 to +1 . The value gradually approaches -1 as the alpha wave increases, taking the point $(-1,-1)$ as the origin. The IBRS increases when the $(\mathrm{x}, \mathrm{y})$ coordinate becomes close to this origin. The vector length from the origin is defined as the IBRS, as shown in Fig.2. We estimate the human state by using this index.

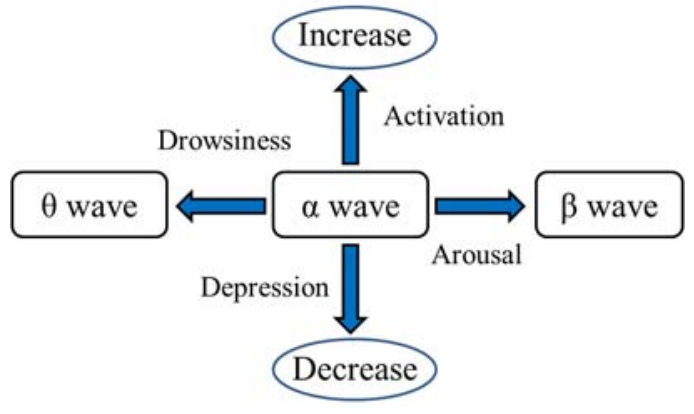

Fig.1 Relationship between the human state and brainwave frequencies

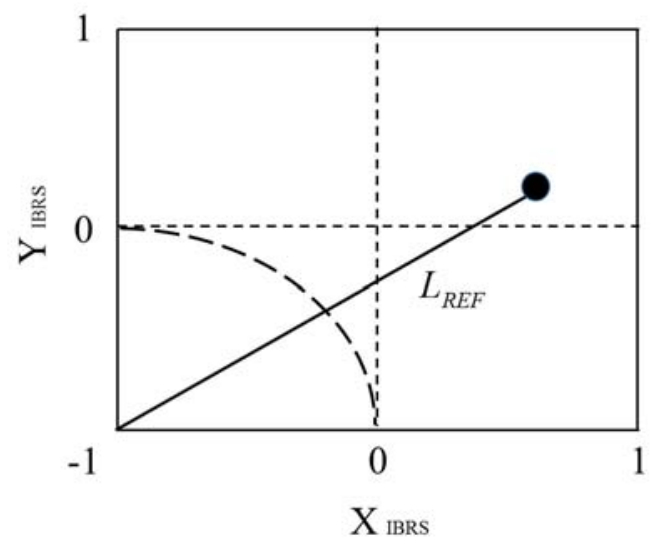

Fig.2 2D display of the IBRS

$$
\begin{gathered}
X_{I B R S}=\frac{\theta_{s t r}-\alpha_{s t r}}{\theta_{s t r}+\alpha_{s t r}} \\
Y_{I B R S}=\frac{\beta_{s t r}-\alpha_{s t r}}{\beta_{s t r}+\alpha_{s t r}}
\end{gathered}
$$




\subsection{Fabrication of an illumination system with color rendering functionality}

This section describes the fabrication of a system that automatically controls the color spectra of LED lamps to improve comfort. A $2.0 \times 1.2 \times 2.1 \mathrm{~m}$ (length $\times$ width $\times$ height) room was designed to block outside light (Fig. 3). Red (R), green (G), and blue (B) LEDs capable of emitting monochromatic color were installed on the ceiling of the room. A high-illuminance $6 \mathrm{~W}$ RGB LED manufactured by OptoSupply Inc. was used to set maximum illuminance to approximately $420 \mathrm{~lx}$. Because the brightness of ordinary classrooms is designed to be 500 $1 \mathrm{x}$ or more, it is thought that designed LEDs have enough lighting capability for the comfortable space. The LED lamps were tightly adhered to a $250 \times 600 \times 5 \mathrm{~mm}$ (length $\times$ width $\times$ thickness) aluminum plate to emit the heat. The aluminum plate was installed on the ceiling of the room. The wavelengths of the LED lamps were $\mathrm{R}=624 \mathrm{~nm}, \mathrm{G}=$ $525 \mathrm{~nm}$, and $\mathrm{B}=460 \mathrm{~nm}$. The brightness and color in the room could be controlled by adjusting the current to the red, green, and blue LEDs. To realize the functionality above, a drive circuit was designed and fabricated. Fig. 4 shows the appearance of the fabricated LED drive circuit. Ammeters are equipped on the front panel of the drive circuit to adjust the color of each LED accurately. The color of LEDs tend to drift slightly over time. To prevent this inconvenience, the LED drive circuit has a feedback system to observe the current. For automatic control, brightness and color are controlled by the signal transmitted from a microcomputer (Arduino via Bluetooth) to the LED drive circuit.

\subsection{Signal flow of the illumination system}

Fig. 5 shows the construction of the illumination system. First, the subject wearing the EEG sensor enters the room. Then, the illumination system performs the check process for a few minutes to find a calming color for the subject. During this time, the EEG sensor keeps transmitting brainwave signals to the Arduino. Using the Arduino, the IBRS is calculated using the brainwave signals and comfort is estimated accordingly. After this process, the calming color is decided based on the estimation procedure described below and the system illuminates the room using this color.

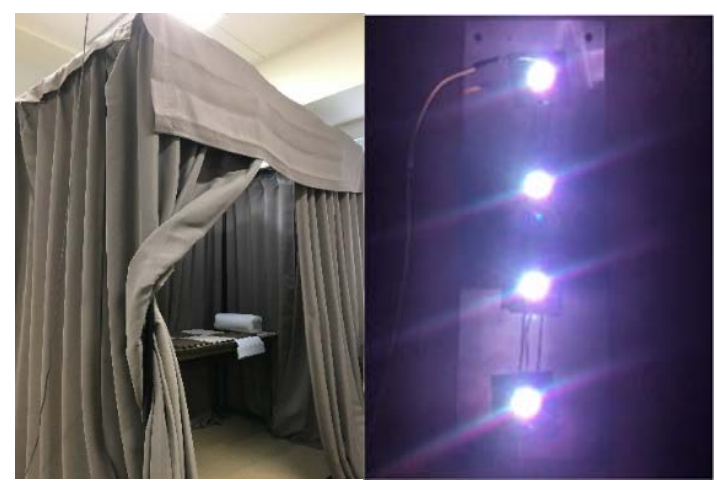

Fig.3. Appearance of the (1) darkroom, (2) installed red, green, and blue LEDs

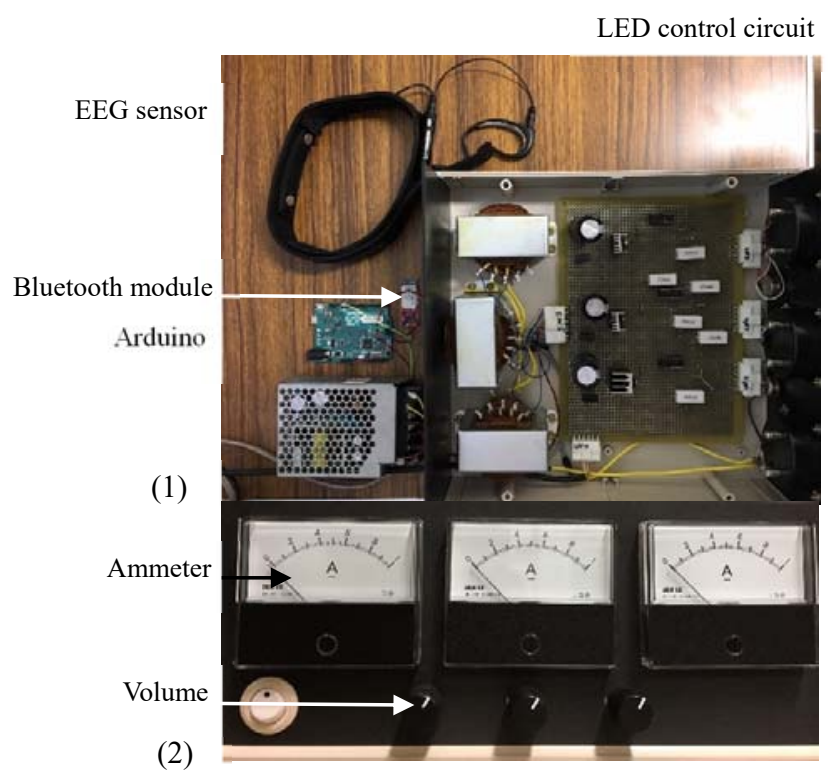

Fig.4 Fabricated LED drive circuit

(1) all components, (2) front panel

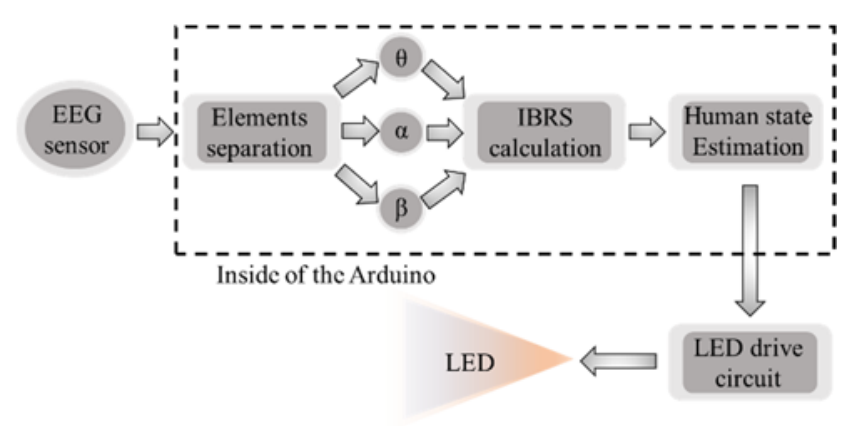

Fig.5 Configuration of designed illumination System 


\subsection{Estimation method of the calming color}

It is difficult to select a specific calming color because red, green, and blue LEDs can emit many colors. In this study, the calming color was selected in the following way. First, the luminosity of the red, green, and blue LEDs was adjusted to the same level to be used as a baseline. The spectra of LEDs were changed from this state during the estimation process. An MK-350N handheld spectrometer manufactured by CURtek corp was used to measure the wavelength and luminosity of the LEDs. From here, only the red component was changed for three steps; the measurement time per step is one minute. After that, the red component was set back to the baseline. Next, the green component was similarly changed. This time, because the baseline has the same red, green, and blue components, this can be done in two steps. Because the remaining blue component is measured in the same way, the EEG signals for all 7 steps take $7 \mathrm{~min}$ to collect. The average value of each interval of the IBRS are calculated after the estimation process, and the color estimated to be the most calming is determined using the following method. First, intervals having the IBRS over 1.0 are eliminated. The standard deviations of intervals of candidates are compared in the next step. The interval with the lowest standard deviation is determined as the resting state, and the system illuminates the room with the color of the interval. If there is no interval with an IBRS under 1.0, then the system determines that no resting color existed during the 7 intervals.

\section{Result and discussion}

Fig. 6 shows the measured IBRS for the changing colors over the 7 intervals. The three sets of data in Fig. 6 were obtained from experiments conducted using the same subject on different dates. Fig. 7 shows relative luminosity with respect to the wavelength of red, green, and blue light in each interval. Fig. 8 shows the average IBRS and standard deviation for each interval. Table 1 lists the intervals arranged in descending order of average IBRS. Shaded areas in table 1 represent the intervals with an IBRS lower than 1.0. Although the values of the third and fifth interval in the second iteration seem to be the same, there were slight differences after the decimal point. The measured BRSIs shown in Fig. 6 followed roughly the same response pattern to the emitted colors in each interval of the three experiments. Among the measured intervals, the two intervals with the lowest values were the third and fifth intervals in all three experiments. Although the light components in the third and fifth intervals shown in Fig. 7 were not similar, the naked eye classified them as was white.

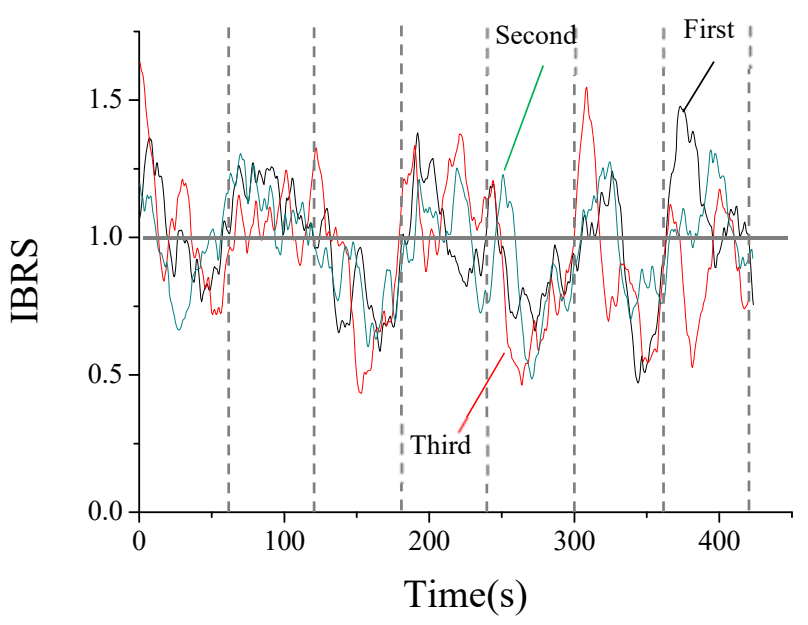

Fig.6. Measured IBRS for the changing color of 7 intervals

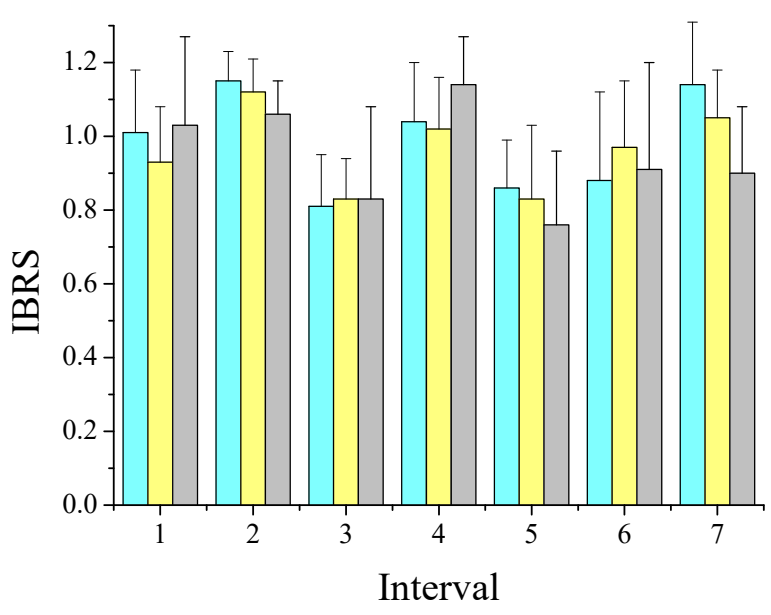

Fig. 8 Average value and standard deviation of the IBRS

Table.1 Descending order of average value of the IBRS

\begin{tabular}{|c|c|c|c|c|c|c|c|}
\hline First time & 3 & 5 & 6 & 1 & 4 & 7 & 2 \\
\hline SD & 0.14 & 0.13 & 0.24 & 0.17 & 0.16 & 0.17 & 0.08 \\
\hline Second time & 3 & 5 & 1 & 6 & 4 & 7 & 2 \\
\hline SD & 0.11 & 0.2 & 0.15 & 0.18 & 0.14 & 0.13 & 0.09 \\
\hline Third time & 5 & 3 & 7 & 6 & 1 & 2 & 4 \\
\hline SD & 0.2 & 0.25 & 0.18 & 0.29 & 0.24 & 0.09 & 0.13 \\
\hline
\end{tabular}




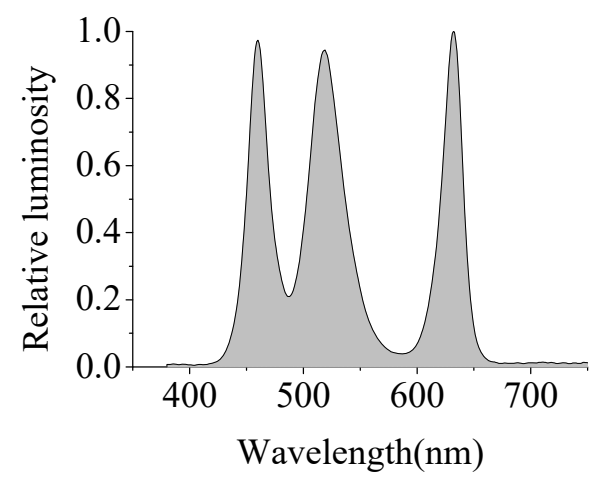

(1) First section

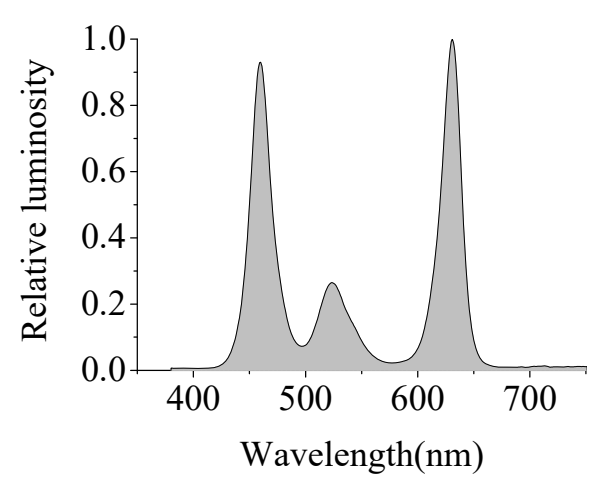

(2) Second section

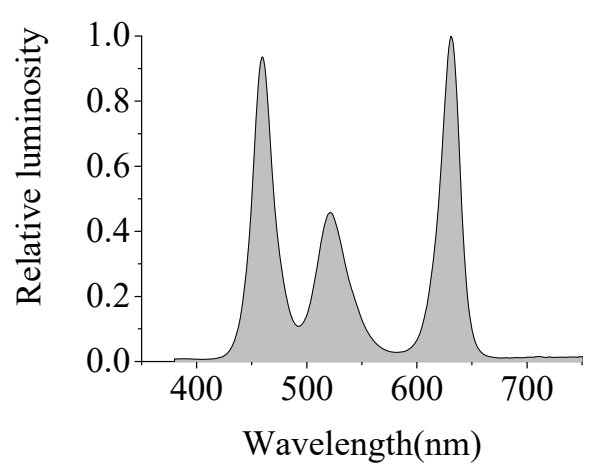

(3) Third section

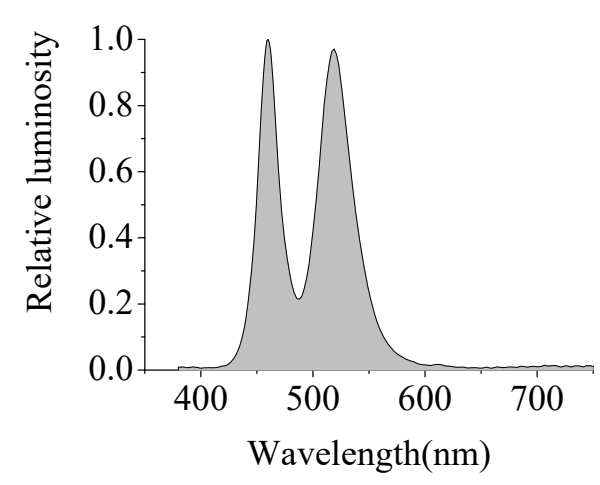

(4) Fourth section

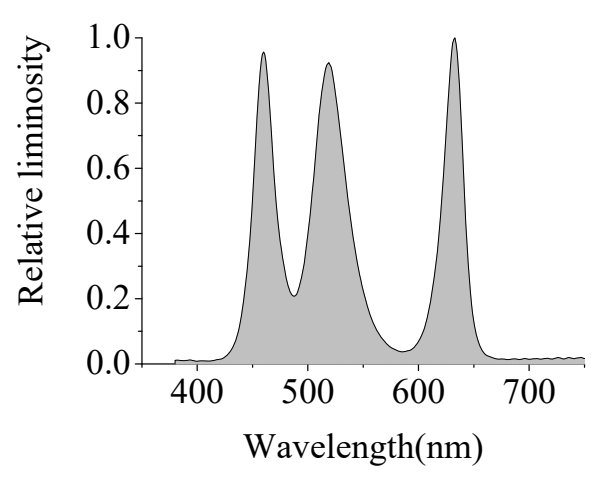

(5) Fifth section

(6)
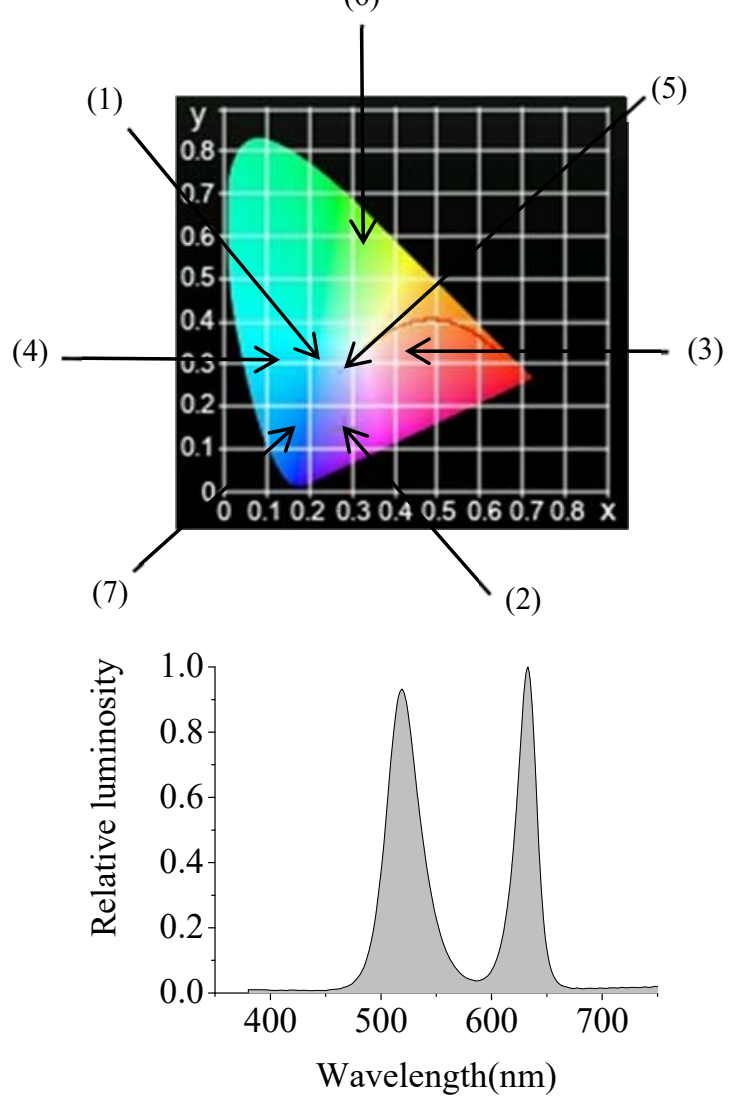

(6) Sixth section

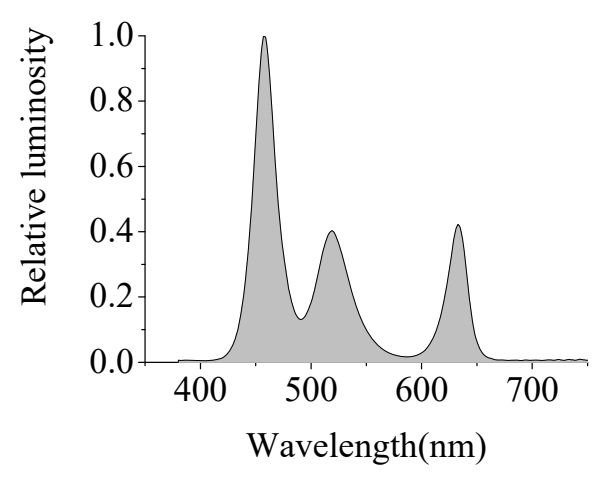

(7) Seventh section

Fig.7 Relative luminosity for the wavelength of red, green, and blue in each interval. 
Considering only the average value, these two colors were the most calming. However, it is necessary to consider the movement of the IBRS during the interval in addition to the average. Even if the average IBRSs were almost same, comfort might differ depending on if EEG fluctuated widely or remained steady. Thus, standard deviation is considered next. A low the standard deviation means that data in the interval is stably plotted around the average. For intervals with an IBRS lower than 1.0 in one to three experiments, the intervals with the lowest standard deviation were, in ascending order, the fifth, third, and seventh (Fig. 8). The color of each interval is shown in Fig. 7. Therefore, the system estimated these colors to be the most calming and illuminated the room with these colors. From these results, the calming effect of colors can be determined using EEG data, although further research is needed to understand if the subjects actually prefer these colors. However, an illumination system that estimates human comfort using EEG signals and adjust to a calming color on an individual basis is realizable.

\section{Conclusion}

In this study, we proposed an LED illumination system that automatically estimates human comfort by capturing and analyzing brainwaves in real time and adjusts the brightness and color of an LED lamp to improve environmental comfort. To realize this proposal idea, an illumination system consisting of a darkroom, LED drive circuit (including red, green, blue LEDs), and microcomputer was designed and fabricated. Human comfort was estimated using the IBRS calculated by analyzing each frequency element from measured brainwaves. Three types of data obtained from experiments conducted by one subject on different dates were analyzed. Colors with an average IBRS value less than 1.0 were extracted first, and then standard deviation was checked. Afterward, the system estimated the most calming color. Calm states, as induced by LED color, could be determined from the EEG results. Therefore, a system that estimates human comfort using EEG signals and adjusts to a calming color on an individual basis is realizable.

\section{References}

(1) T.KOTANI, "Spectral Power Distributions with Color Appearance of LED Light Sources", Toshiba review, Vol.65, No.4, pp.68-71, 2010.
(2) T.KOTANI, H.HIGASHI, “Approach for Realization of Comfortable Lighting Environment Using LED Luminaires", Toshiba review, Vol.65, No7, pp.20-23, 2010.

(3) B.w.KO, D.g.LEE, H.c.CHIANG, T.KOGA, and K.HIRATE, "BASIC STUDY ON REST EFFECT OF LIGHT COLOR IN THE LED LIGHTING”, J.Environ.Eng, Vol.76, No.662, pp.363-368, Apr, 2011.

(4) H.Kubo, Y.Inoue, "The Influence of Lighting by Used Chromatic Light on Physiological Responses", Journal of The Illuminating Engineering Institute of Japan, Vol. 92, No.9, pp.645-649, Sep, 2008.

(5) Y.INOUE, M.TOMARI, "The influence of the chromatic light on visibility, psychophysiological responses", Architectural Institute of Japan, Vol.47, pp.73-76, 2007.

(6) T.Ito, E.Koyama, "The Recent Lighting of the Medical/Welfare Institution Considering Human Circadian Rhythm", J.Illum. Engng. Inst. Jpn, Vol.84, No.6, pp.362-367, 2000.

(7) T.Nishimura, "Lighting Control in Consideration of Circadian Rhythms”, J.IEIE Jpn, Vol.33, No.1, pp.34-36, Jan, 2013.

(8) NeuroSky, "MindSet Communications Protocol", NeuroSky application note (2011).

(9) On the subject of brainwave : digital medic Inc, http://www.digital-medic.com/waves

(10)K.YOSHII and K.Matsumoto, "Estimation of the Human State Based on the Index of Brain Resting State (IBRS) by Using Single-Channel Dry EEG Sensor", ISTS 2015, Aug, 2015.

(11)K.Yoshii and K.matsumoto, "A Dry-Eye Warning System Based on the Estimation of the Human State Using a Single-Channel EEG Sensor", ICISIP 2016, pp.142-148, Sep, 2016.

(12) K.Fujimoto and K.matsumoto, "Sleep Support System for Providing Comfortable Sleep Environment Using a Single-Channel EEG Sensor”, ICISIP2017, pp.146-151, Sep, 2017.

(13) T.Ichikawa, "An Invitation to Journey for Understanding of EEG", Seiwa Shoten Publishers, 2006, in Japanese. 